\title{
Lifetimes of Circumstellar Disks in Lynds 1641
}

\author{
Lori E. Allen ${ }^{1,2}$ \\ School of Physics, University of New South Wales, Sydney, NSW, 2052, \\ Australia \\ Karen M. Strom ${ }^{1}$ \\ Department of Physics and Astronomy, University of Massachusetts, \\ Amherst, MA 01002
}

\begin{abstract}
It is by now evident that most young stars have associated disks and/or envelopes, which may be active (accreting) or passive (reprocessing stellar photons), or both. Knowing how such disks evolve is crucial to our understanding of how stars form, and may be relevant to other questions, such as the time available for the formation of planets in solar nebulae. In this poster we discuss the properties of pre-main sequence circumstellar disks in Lynds 1641 in Orion, the nearest giant molecular cloud complex.
\end{abstract}

\section{Introduction}

Based on their optical (R,I) and infrared $(\mathrm{J}, \mathrm{H}, \mathrm{K})$ imaging survey, Strom, Strom, \& Merrill (1993) demonstrated that $60 \%$ of the closely grouped, obviously young "aggregate" stars in Lynds 1641 have active accretion disks. Here we employ spectroscopic and photometric data to examine the frequency of circumstellar disks in L1641, as a function of stellar age and mass. Stellar age and mass are estimated by placing stars in the H-R diagram and comparing their locations with pre-main sequence evolutionary tracks (D'Antona \& Mazzitelli 1994). Disks are detected by comparing extinction-corrected $(\mathrm{H}-\mathrm{K})$ colors of stars with their intrinsic colors, as indicated by spectral type. Stars displaying a $3 \sigma$ excess in $(\mathrm{H}-\mathrm{K})$ are said to be disked, and are represented as filled symbols in Figure 1.

\section{Summary}

1. The disk frequency among young stars in L1641 is $50 \%-80 \%$.

2. Although the disk frequency is lower for older stars, there is evidence that several stars have retained their disks for 3-10 Myr.

\footnotetext{
${ }^{1}$ Visiting Astronomer, Kitt Peak National Observatory. KPNO is operated by AURA, Inc. under cooperative agreement with the National Science Foundation

${ }^{2}$ Department of Physics and Astronomy, University of Massachusetts, Amherst, MA 01003
} 


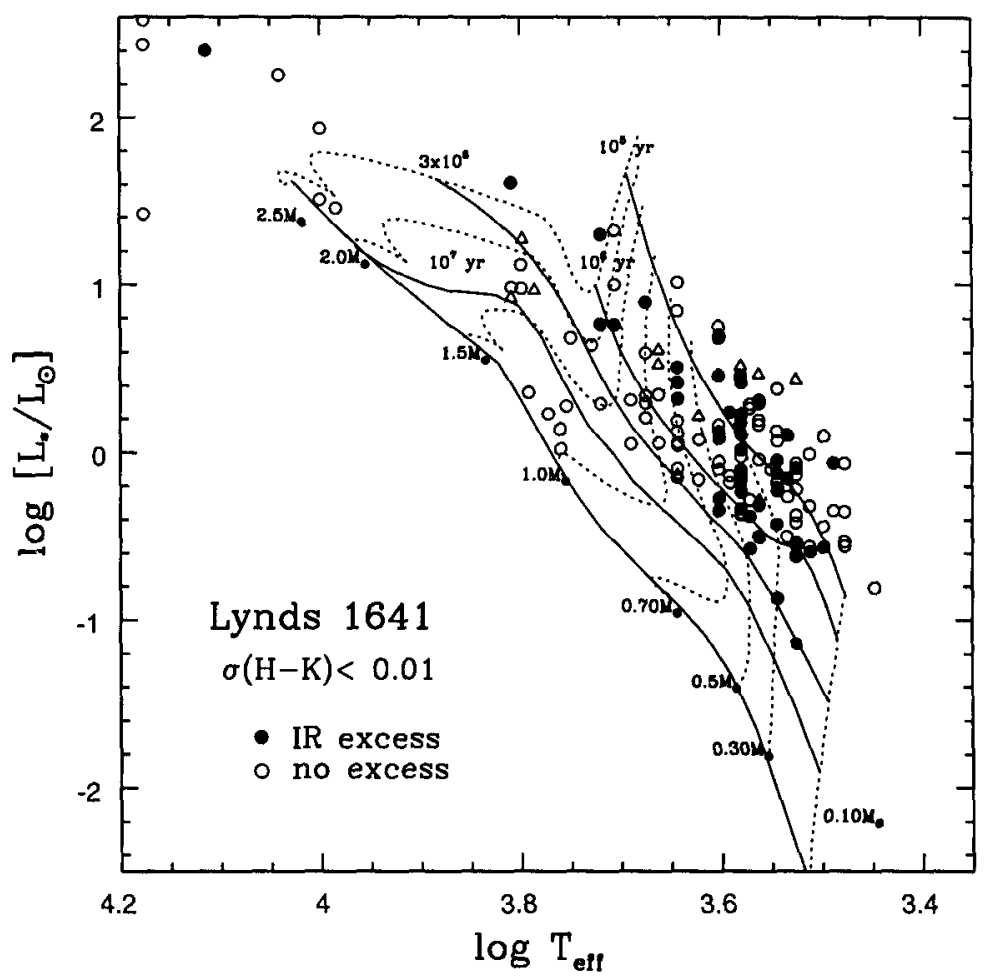

Figure 1. HR diagram of pre-main sequence stars in Lynds 1641

3. The disk frequency is strongly dependent on stellar mass, in the sense that it is higher (x2) for low-mass stars than for high-mass stars (Table 1).

Table 1. Disk fraction as a function of stellar mass

\begin{tabular}{cc}
\hline Mass Range & Disk fraction (\%) \\
\hline $\mathrm{M}>1 \mathrm{M} \odot$ & 14 \\
$0.5 \leq \mathrm{M} \leq 1.0 \mathrm{M}_{\odot}$ & 24 \\
$\overline{\mathrm{M}}<0.5 \mathrm{M}_{\odot}$ & $\mathbf{4 2}$ \\
\hline
\end{tabular}

\section{References}

D'Antona, F. \& Mazzitelli, I. 1994, ApJS, 90, 467

Strom, K.M., Strom, S.E., \& Merrill, K.M. 1993, ApJ, 412, 233 